\title{
Effets de différents modes de labour sur le rendement et la rentabilité de la culture du manioc (Manihot esculanta Crantz) à M'vuazi, RD Congo
}

\author{
Tony Muliele MUKU ${ }^{1,2 *}$, Philippe Mampasi MBUNGU² et \\ Emmanuel Bambala NKULUKUTA ${ }^{2}$ \\ ${ }^{1}$ Université Pédagogique Nationale (UPN), Faculté des Sciences Agronomiques. B.P. 8815, Kinshasa \\ Ngaliema, RD Congo. \\ ${ }^{2}$ Institut National pour l'Etude et la Recherche Agronomiques (INERA). B.P. 2037, Kinshasa Gombe, \\ RD Congo. \\ *Auteur correspondant ; E-mail : tonymuliele@yahoo.fr; Tél : (+243) 853158822.
}

\section{RÉSUMÉ}

Les connaissances sur les modes de labour sont nécessaires en vue d'identifier la méthode de labour appropriée pour améliorer le rendement des cultures dans une zone agro écologique spécifique. Cette étude visait à évaluer l'effet des modes de labour sur le rendement et la rentabilité de la culture du manioc (Manihot esculanta Crantz, cultivar "Mayombe") à M'vuazi. Trois traitements à savoir : labour à plat ou contrôle ( $\left.\mathrm{T}_{0}\right)$, labour en billon $\left(\mathrm{T}_{1}\right)$ et labour en butte $\left(\mathrm{T}_{2}\right)$ ont été comparés sous un dispositif expérimental en blocs complets randomisés avec 4 répétitions. A la récolte, le nombre des racines tubéreuses commerçables, le rendement en racines tubéreuses, le rendement en pulpe fraîche et le rendement en matière sèche ont été mesurés. L'analyse coût-bénéfice a été appliquée pour évaluer la rentabilité des modes de labour. Le nombre de racines tubéreuses par plante était de 8,7 sous $\mathrm{T}_{0}, 8,9$ sous $\mathrm{T}_{1}$ et 9,6 sous $\mathrm{T}_{2}$. Les traitements $\mathrm{T}_{0}$ et $\mathrm{T}_{2}$ ont donné $40 \mathrm{t} \mathrm{ha}^{-1}$ des racines tubéreuses contre $37,5 \mathrm{t} \mathrm{ha}^{-1}$ sous $\mathrm{T}_{1}$. Le $\mathrm{T}_{2}$ a donné le rendement en pulpe fraîche le plus élevé suivi de $\mathrm{T}_{0}$ et $\mathrm{T}_{1}$ tandis que le rendement en matière sèche était similaire dans tous les traitements. Toutefois, pour tous les paramètres mesurés, l'analyse statistique n'a pas révélé de différences significatives entre les traitements. L'analyse coût-bénéfice a révélé un manque à gagner de 288 pour $\mathrm{T}_{1}$ et 88 SUS pour $\mathrm{T}_{2}$ par rapport à $\mathrm{T}_{0}$. Dans les conditions agro écologiques de cette étude, le labour en billon et en butte semblent donc être inopportuns mais nécessitent d'être évalués sur d'autres types de sols et des terrains accidentés.

(C) 2020 International Formulae Group. All rights reserved.

Mots clés : Travail du sol, manioc, rendement, analyse coût-bénéfice, M’vuazi.

\section{Effects of different tillage modes on yield and profitability of cassava (Manihot esculanta Crantz) at M'vuazi in DR Congo}

\begin{abstract}
The data on tillage modes are needed in order to identify appropriate tillage methods to improve crops yield in a specific agro ecological zone. Hence, three tillage modes including flat tillage ( $T_{0}$ or control), mound tillage $\left(T_{1}\right)$, and ridge tillage $\left(T_{2}\right)$ were compared as to their effects on cassava (Manihot esculanta Crantz, "Mayombe" cultivar) yield and related-profitability at M'vuazi in DR
\end{abstract}


Congo. Field layout design was the randomized complete block design with three above-mentioned treatments and four repetitions. Tillage in all treatments was done using a hand hoe. At harvest (11 months after planting), the number of marketable tubers per plant, cassava fresh tubers yield, cassava fresh flesh yield and cassava dry matter tubers yield were measured. Cost-benefit analysis was computed in order to evaluate the profitability of tillage modes types. Tillage modes do not significantly affect cassava yield and profitability. However, the number of marketable tubers per plant was slightly higher under $\mathrm{T}_{2}$ (9.7 roots plant ${ }^{-1}$ vs 8.7 on $\mathrm{T}_{0}$, and 8.9 on $_{1}$ ). $\mathrm{T}_{0}$ and $\mathrm{T}_{2}$ yielded 40 t ha ${ }^{-1}$ of cassava fresh tubers yield, and $37.5 \mathrm{tha}^{-1}$ on $\mathrm{T} 1$ treatment. Cassava fresh flesh yield decreased following this order: $\mathrm{T}_{2}\left(30.3 \mathrm{tha}^{-1}\right)>\mathrm{T}_{0}\left(29.1 \mathrm{tha}^{-1}\right)>\mathrm{T}_{1}\left(27.3 \mathrm{t} \mathrm{ha}^{-1}\right)$, whereas cassava dry matter tubers yield was similar across treatments. Compared to $\mathrm{T}_{0}$ (control), cost-benefit analysis revealed a shortfall of 288 and 88 USD (US dollars) in $\mathrm{T}_{1}$ and $\mathrm{T}_{2}$ treatments, respectively. We conclude that mound $\left(T_{1}\right)$, and ridge $\left(T_{2}\right)$ tillages seem to be inappropriate to improve cassava yield in the study area, but need to be evaluated on others soil types and fields on slope.

(c) 2020 International Formulae Group. All rights reserved.

Keywords: Plowing, cassava, yield, cost-benefit analysis, M'vuazi.

\section{INTRODUCTION}

Le manioc (Manihot esculanta Crantz) est l'une des principales cultures en République démocratique du Congo (RD Congo) en général, et en province du Kongo Central en particulier. Il y est cultivé pour ses racines tubéreuses, et ses feuilles qui entrent principalement dans l'alimentation humaine (HarvestPlus, 2010 ; Mahungu et al., 2015; Mondo et al., 2019). A l'échelle nationale, le manioc couvre environ $50 \%$ des terres emblavées (HarvestPlus, 2010) et occupe la première position en termes des quantités produites et des revenus générés (FAO, 2014). La consommation annuelle du manioc par habitant en RD Congo est la plus élevée au monde et est évaluée à environ $353 \mathrm{~kg}$ (HarvestPlus, 2010).

La production du manioc en RD Congo est essentiellement assurée par de petits producteurs pour l'autosubsistance. En effet, du fait de sa plasticité à produire sur des terrains à faible fertilité (Temegne et al., 2015) et de la flexibilité des dates de récolte, le manioc est une culture de soudure de premier choix pour les agriculteurs de plusieurs pays producteurs du manioc à travers le monde (Bilong et al., 2017 ; Mondo et al., 2019).

$\mathrm{Au}$ début des saisons culturales ("saisons A"'- octobre et "saison B"-- mars), le sol est préparé suivant différents modes du travail du sol avant la plantation de boutures de manioc. Suivant le précédant cultural (forêt ou jachère herbacée) et les moyens financiers du producteur, ce dernier recourt au non labour (plantation directe après déboisement pour le cas de forêt) ou au labour qui est soit manuel, soit mécanisé au moyen du tracteur (Fasinmirin et Reichert, 2011). Il a été constaté qu'en milieu paysan en RD Congo et bien d'autres pays tropicaux, le labour est principalement manuel, et est fait soit à plat, soit en billon ou en butte (Fasinmirin et Reichert, 2011).

Des études antérieures ont rapporté que les modes de préparation du sol et les pratiques culturales influencent le rendement du manioc. Sur un sol sablo-argileux, Mahungu et al. (2015) ont rapporté un accroissement de rendement de $45 \%$ sur les parcelles labourées comparativement aux parcelles non labourées. Cependant, ces auteurs ont également observé que le travail du sol n'affectait pas le rendement du manioc sur le sol sableux du Plateau des Batékés (RD Congo). Kupukula et Mwangu (2017) ont observé que, comparativement au labour à plat sans enfouissement de paille, le labour en billon avec enfouissement de la paille $\left(5 \mathrm{t} \mathrm{ha}^{-1} \mathrm{de}\right.$ paille de Loudetia simplex) accroissait le rendement du manioc sur le sol sableux de Kiyaka (RD Congo). Lamadi (2016) a évalué l'effet de différents modes de labour (non labour, labour minimum et le labour conventionnel) sur le rendement du manioc 
avec un sol sable limoneux au Nigeria. L'auteur a rapporté le rendement le plus élevé sur les parcelles soumises au labour conventionnel, tandis que les parcelles non labourées ont donné le rendement le plus faible. Au Ghana, des résultats similaires ont été rapportés (Fasinmirin et Reichert, 2011). Au Zaïre (RD Congo), ces auteurs ont rapporté des résultats contradictoires sur l'effet du labour et du paillage sur le rendement du manioc : (i) des rendements similaires entre les parcelles labourées et les parcelles non labourées sur sol à texture limon argilosableux, (ii) des rendements supérieurs sur des parcelles non labourées par rapport aux parcelles labourées sur un oxisol, (iii) des rendements plus faibles sur des parcelles non labourées avec ou sans paillage comparativement aux parcelles labourées.

Par ailleurs, tout bien considéré, si tous ces modes de préparation du sol ont tous un même objectif (préparer le lit de semis/plantation et détruire les adventices), ils n'ont cependant pas le même coût. Conséquemment, toute chose restant égale par ailleurs, le mode de préparation du sol le moins onéreux et permettant d'obtenir le rendement le plus élevé serait la meilleure option en vue d'améliorer le revenu des producteurs du manioc dans une région de production donnée.

Ainsi, dans la perspective du choix de la meilleure option de préparation du sol en conditions paysannes (milieu réel), l'objectif de cette étude est donc d'évaluer l'effet de trois (3) modes de labour, notamment le labour à plat, le labour en billon et le labour en butte sur le rendement et la rentabilité du manioc en conditions agro écologiques de M’vuazi.

\section{MATÉRIEL ET MÉTHODES}

Cette étude a été conduite à M'vuazi $\left(5,451{ }^{\circ} \mathrm{S}, 14,893{ }^{\circ} \mathrm{E}, 427 \mathrm{~m}\right.$ d'altitude), un centre de recherche de l'Institut National pour l'Etude et la Recherche Agronomiques (INERA, RD Congo). Suivant la classification de Köppen, le climat de M'vuazi est de type Aw4 (climat tropical avec 4 mois de saison sèche). La distribution annuelle des pluies est bimodale ("saison A"et "saison B"). La saison culturale à commencé en octobre et se clôture en février tandis que la saison culturale $\mathrm{B}$ couvre la période allant de mars à mai. La pluviométrie moyenne annuelle varie entre 1 400 et $1600 \mathrm{~mm}$ (Muliele et al., 2018 ; Yuku et Kimbembi, 2018). La Figure 1 présente l'évolution de la pluviosité et de la température au cours de la période de l'expérimentation. Cette figure montre que la culture a bénéficié de $1517 \mathrm{~mm}$ de pluie (en moyenne $126 \mathrm{~mm}$ de pluie par mois), et la température a oscillé entre 22,8 et $26,5^{\circ} \mathrm{C}$. Les sols de M'vuazi n'ont pas encore été décrits dans les détails ces dernières années, les caractéristiques disponibles datent des années 50. Selon Denisoff et Devred (1954), il s'agit des sols alluvionnaires formés sur l'esquisse géologique d'argile résiduelle. Ils présentent de bonnes propriétés (fertilité et résistance à l'érosion). On leur reproche néanmoins la superficialité de la nappe phréatique et le risque d'inondation. Le Tableau 1 présente quelques caractéristiques physico-chimiques du sol du site d'expérimentation, et des environs (Kabongo et al., 2019).

L'essai a été installé en octobre 2017 ("saison A") sur un terrain dont le précédant cultural était la jachère à Pennisetum purpureum. Le terrain expérimental était quasiplat (pente inférieure à 5\%). La végétation était fauchée à la machette avant le travail du sol à l'aide d'une houe manuelle. Les traitements appliqués consistaient en trois modes de labour : le labour à plat ou contrôle $\left(\mathrm{T}_{0}\right)$, le labour en billon $\left(T_{1}\right)$ et le labour en butte $\left(T_{2}\right)$. Le dispositif expérimental était le bloc complet randomisé à 3 traitements susmentionnés et quatre répétitions. Chaque bloc comportait 4 parcelles élémentaires de $4 \mathrm{~m}$ x $3 \mathrm{~m}$ séparées de $1 \mathrm{~m}$.

Le matériel végétal planté était constitué des boutures de manioc de la variété « Mayombe » dont les principaux caractères agronomiques sont : une durée à la maturité de 12 à 15 mois, 3 à 5 racines tubéreuses par plant, un rendement en tubercules de l'ordre 30 à 40 $\mathrm{t} \mathrm{ha}^{-1}$ en conditions contrôlées et 12 à $15 \mathrm{tha}^{-1}$ en milieu paysan. Quant aux exigences écologiques, cette variété de manioc exige un sol de bonne qualité et riche en matière 
organique. Elle peut croître en zones d'altitude jusqu'à 1730 m d'altitude (MINAGRI, 2012).

Les boutures, fragments de $20 \mathrm{~cm}$ des tiges saines de manioc, ont été plantées aux écartements recommandés par l'Institut National pour l'Etude et la Recherche Agronomiques (INERA, RD Congo) pour la production des racines tubéreuses : $1 \mathrm{~m} \times 1 \mathrm{~m}$, soit une densité de plantation de 10000 plants ha $^{-1}$. La reprise a commencé 6 jours après plantation et était complète au dixième jour. L'entretien a concerné essentiellement le sarclage, 3 sarclages au total ont été effectués. La pression de mauvaises herbes était identique dans tous les traitements. Aucun engrais minéral ni amendement calcique n'a été appliqué, de même le contrôle phytosanitaire n'a pas été effectué du fait de l'absence des symptômes des maladies.

Le rendement a été évalué à la récolte qui est intervenue en octobre 2018, soit 11 mois après la plantation (MAP). Pour chaque parcelle élémentaire, le nombre de racines tubéreuses des plantes du «net plot»a été déterminé en dénombrant les racines commerçables. La masse des racines tubéreuses (pulpe + épiderme) a été mesurée à l'aide d'une balance (marque SALTER, poids max. $25 \mathrm{~kg}$ ), immédiatement après récolte ; et extrapolée à l'hectare pour obtenir le rendement $\left(\mathrm{t} \mathrm{ha}^{-1}\right)$ en racines tubéreuses. Un échantillon des racines tubéreuses a été prélevé pour déterminer le rendement en pulpes (tonne de pulpe fraîche $h^{-1}$ ) et le rendement en matière sèche (tonne de matière sèche ha ${ }^{-1}$ ). $\mathrm{Ce}$ dernier rendement a été obtenu par la différence entre la masse de pulpe avant (masse fraîche) et après séchage (masse sèche) à l'étuve à $65{ }^{\circ} \mathrm{C}$ pendant $72 \mathrm{~h}$. Les données collectées (nombre de racines tubéreuses par plante, rendement en racines tubéreuses, rendement en pulpe fraîche et rendement en matière sèche) ont été analysées au moyen du logiciel SPSS 20. L'analyse statistique a concerné la statistique descriptive (moyenne et erreur standard) et l'analyse de la variance au seuil de probabilité de 0,05 . L'analyse coûtbénéfice a été appliquée pour évaluer la rentabilité de différents modes de préparations du sol étudiés. Le coût de production a concerné les dépenses de préparation du sol, d'achat des semences, de plantation, d'entretien et de récolte. Le revenu, par contre, a été évalué sur base du rendement en racines tubéreuses (pulpe + épiderme), soit les racines fraîchement récoltées et vendues sans transformation préalable.

Tableau 1 : Caractéristiques physico-chimiques du sol du site expérimental $(0-20 \mathrm{~cm})$.

\begin{tabular}{lc}
\hline Propriétés du sol & Valeurs \\
\hline $\mathbf{p H}\left(\mathbf{H}_{\mathbf{2}} \mathbf{O}\right)$ & 5,60 \\
Carbone organique (\%) & 2,19 \\
$\mathbf{C a}(\mathbf{p p m})^{\mathbf{1}}$ & 1505 \\
$\mathbf{M g}(\mathbf{p p m})^{\mathbf{1}}$ & 229 \\
$\mathbf{K}(\mathbf{p p m})^{\mathbf{1}}$ & 105 \\
$\mathbf{C E C}(\mathbf{m e ́ q} / \mathbf{1 0 0 g})^{\mathbf{1}}$ & 13,76 \\
$\mathbf{P}$ assimilable $(\mathbf{p p m})$ & 32,20 \\
\hline \multicolumn{1}{c}{ 1. Source : Kabongo et al. (2019) }
\end{tabular}




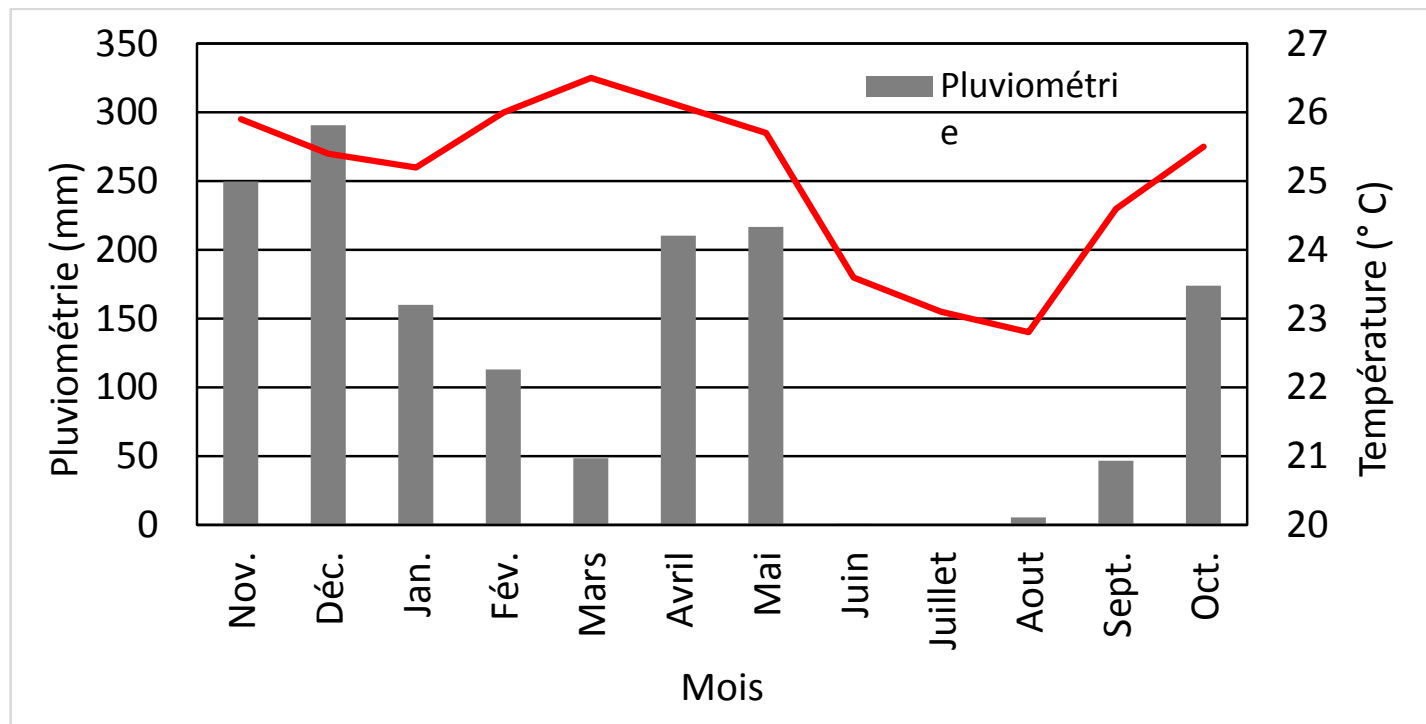

Figure 1 : Evolution de la pluviosité et de la température au cours de la période de l'expérimentation. Source : Service de météorologie, Antenne GCRN, INERA M'vuazi.

Tableau 2: Paramètres de rendement du manioc (11 MAP) suivant les différents modes de préparation du sol à M'vuazi. Valeurs = moyennes $(n=4) \pm$ erreurs standards.

\begin{tabular}{lcccc}
\hline Traitements & $\begin{array}{c}\text { Nombre de RT } \\
\text { plant }^{-1}\end{array}$ & $\begin{array}{c}\text { Rendement PF } \\
\left(\mathbf{t ~ M F h a ~}^{-1}\right)\end{array}$ & \% MS & $\begin{array}{c}\text { Rendement MS } \\
\left.\text { (t MSha }^{-1}\right)\end{array}$ \\
\hline Labour à plat $\left(\mathbf{T}_{\mathbf{0}}\right)$ & $8,9 \pm 1,1$ & $29,1 \pm 4,1$ & $40,0 \pm 0,8$ & $11,7 \pm 1,7$ \\
Labour en billon $\left(\mathbf{T}_{\mathbf{1}}\right)$ & $8,7 \pm 0,7$ & $27,3 \pm 0,5$ & $41,5 \pm 0,8$ & $11,3 \pm 0,4$ \\
Labour en butte $\left(\mathbf{T}_{\mathbf{2}}\right)$ & $9,6 \pm 1,4$ & $30,3 \pm 3,4$ & $38,6 \pm 1,0$ & $11,7 \pm 1,2$ \\
\hline
\end{tabular}
RT : racines tubéreuses, $\mathrm{PF}$ : pulpe fraîche, MS : matière sèche.

Tableau 3: Analyse coût-bénéfice de la culture du manioc (variété 'Mayombe", récoltée 11 MAP) suivant les modes de préparation du sol à M'vuazi.

\begin{tabular}{|c|c|c|c|c|c|c|}
\hline Outputs & $\mathbf{T}_{\mathbf{0}}$ & & $\mathbf{T}_{1}$ & & $\mathbf{T}_{2}$ & \\
\hline Prix unitaire & $\begin{array}{l}\operatorname{Rdt}^{1} \\
\left(\mathrm{t} \mathrm{ha}^{-1}\right)\end{array}$ & $\begin{array}{l}\text { Revenu } \\
\text { (\$) }\end{array}$ & $\begin{array}{l}\text { Rdt } \\
\left(\mathrm{t} \mathrm{ha}^{-1}\right)\end{array}$ & $\begin{array}{l}\text { Revenu } \\
(\$)\end{array}$ & $\begin{array}{l}\text { Rdt } \\
\left(\mathrm{t} \mathrm{ha}^{-1}\right)\end{array}$ & $\begin{array}{l}\text { Revenu } \\
(\$)\end{array}$ \\
\hline $82 \$ t^{-1}$ & 40 & 3280 & 37,5 & 3075 & 40 & 3280 \\
\hline Inputs & $\mathrm{HJ}^{2} \mathrm{ha}^{-1}$ & Coût (\$) & $\mathrm{HJ} \mathrm{ha}^{-1}$ & Coût (\$) & $\mathrm{HJ} \mathrm{ha}^{-1}$ & Coût (\$) \\
\hline 1. Fauchage & 20 & 43 & 20 & 43 & 20 & 43 \\
\hline 2. Préparation du sol & 40 & 86 & 80 & 172 & 80 & 172 \\
\hline 3. Semences & - & 91 & - & 91 & - & 91 \\
\hline 4. Plantation & 10 & 21,5 & 10 & 21,5 & 10 & 21,5 \\
\hline 5. Entretien (3 sarclages) & 100 & 215 & 100 & 215 & 100 & 215 \\
\hline 6. Récolte & 20 & 43 & 20 & 43 & 20 & 43 \\
\hline Total & & 499,5 & & 585,5 & & 585,5 \\
\hline Balance & & 2780,5 & & 2489,5 & & 2694,5 \\
\hline Ratio Bénéfice - coût & & 6,6 & & 5,3 & & 5,6 \\
\hline
\end{tabular}

1. Rdt : Rendement en racines tubéreuses fraiches commerçables (pulpe + épiderme). 2 . HJ : homme-jour $=2,15 \$$, soit 3500 $\mathrm{Fc}$ au taux paritaire de $1 \$ \mathrm{USA}=1630 \mathrm{Fc}$. $\mathrm{T}_{0}$ : labour à plat, $\mathrm{T}_{1}$ : labour en billon, $\mathrm{T}_{2}:$ labour en butte. 


\section{RÉSULTATS}

Le Tableau 2 présente le nombre de racines tubéreuses et le rendement (rendement en pulpe fraîche et rendement en matière sèche) du manioc récolté 11 MAP en fonction des modes de labour. Ce Tableau montre que, quel que soit le paramètre de rendement considéré, les valeurs de différents modes de préparation varient très faiblement. En effet, le nombre de racines tubéreuses par plante s'accroit de 1 seulement dans les parcelles sous labour en butte $\left(\mathrm{T}_{2}\right)$ comparées au labour à plat $\left(\mathrm{T}_{0}\right)$ et labour en billon $\left(\mathrm{T}_{1}\right)$. Le rendement en pulpe fraîche le plus faible est observé dans les parcelles de $\mathrm{T}_{1}\left(27 \mathrm{t} \mathrm{ha}^{-1}\right)$ et augmente de 2 et 3 tonnes de plus dans les parcelles de $\mathrm{T}_{0}$ et $\mathrm{T}_{2}$ respectivement. Cette même tendance a été observée pour le rendement en racines tubéreuses. Contrairement au rendement en pulpe fraîche, la teneur en matière sèche est légèrement supérieure pour les tubercules de $T_{1}$ ( 41,5 contre 38,6 et $40,0 \%$ pour $\mathrm{T}_{2}$ et $\mathrm{T}_{0}$ ) mais n'affecte pas sensiblement le rendement en matière sèche. En dépit de ces tendances, l'analyse de la variance n'a pas révélé de différence significative entre les trois modes de préparation du sol pour tous les paramètres de rendement.

Quant au coût de production, mise à part la préparation du sol dont le coût double pour les parcelles de $T_{1}$ et $T_{2}$, toutes les autres pratiques culturales étaient conduites de la même manière et avaient le même coût (Tableau 3). Aucun traitement n'a affecté de manière significative le revenu (brut ou net). Toutefois, comparés au $\mathrm{T}_{0}$, les traitements $\mathrm{T}_{2}$ et $\mathrm{T}_{1}$ ont entraîné un manque à gagner de 86 et 288 dollars américains (\$US) respectivement.

\section{DISCUSSION}

Le rendement en racines tubéreuses obtenu dans cette étude (37,5 et 40,0 $\mathrm{t} \mathrm{ha}^{-1}$; Tableau 3) correspond bien au rendement rapporté par MINAGRI (2012) pour la variété de «Mayombe». Le nombre moyen des racines tubéreuses par plante de cette étude (89 contre 3-5 racines par plante) est, par contre, supérieur. Les Tableaux 2 et 3 montrent que les modes de préparation du sol n'ont pas affecté de manière significative le rendement du manioc et le revenu y relatif. En d'autres termes, le labour en billon $\left(\mathrm{T}_{1}\right)$ et le labour en butte $\left(T_{2}\right)$ n'ont pas amélioré le rendement du manioc par rapport au labour à plat $\left(\mathrm{T}_{0}\right)$. Comparant, entre autres, le rendement des parcelles labourées et non labourées (Kintché et al., 2017), des parcelles labourées (labour à plat et en billon) et non labourées avec ou sans paillage (Fasinmirin et Reichert, 2011) au Kongo Central-RD Congo ; ces études ont révélé que les modes du travail du sol n'influençaient pas de manière significative le rendement du manioc. Des résultats similaires ont été également rapportés dans d'autres pays (par ex. au Nigeria, FAO, 2013 ; Aiyelari et al., 2002), et d'autres cultures, notamment la patate douce (Agbede et Adekiya, 2009). Des résultats contraires à notre étude ont été cependant rapportés par d'autres auteurs. Ainsi, par exemple, l'étude conduite par Odjugo (2008a) à l'Ouest du Nigéria a démontré un accroissement de 22 et $46 \%$ du rendement de manioc sous labour en billon par rapport au labour en butte et au non labour, respectivement. Pour la culture de l'igname (Dioscorea cayenensis), Odjugo (2008b) a obtenu un rendement significativement supérieur sur les buttes par rapport aux billons et au non labour.

Du point de vue de l'analyse financière, comparé au labour à plat $\left(\mathrm{T}_{0}\right)$, le labour en billon $\left(\mathrm{T}_{1}\right)$ et le labour en butte $\left(\mathrm{T}_{2}\right)$ qui entraînent, respectivement, un manque à gagner de 288 et 88 \$US ha ${ }^{-1}$ semblent inopportuns dans les conditions agro écologiques de notre expérimentation (notamment un terrain quasi uniforme-pente $<5 \%$, sol à fertilité moyenne avec une bonne infiltration, une bonne pluviosité). Toutefois, il importe d'évaluer l'effet de ces différents modes de préparation sur différents types de sol et des terrains accidentés qui sont très fréquents dans la zone d'étude. En effet, les effets des modes de préparation du sol sur le rendement du manioc varient fortement suivant le type de sol, l'histoire du site expérimental et les conditions climatiques pendant la préparation du sol et la plantation (Agbede et Adekiya, 2009; Fasinmirin et Reichert, 2011), et 
peuvent fluctuer d'une année à l'autre (FAO, 2013).

En fin, dans la mesure où les trois (3) modes de labour évalués dans cette étude n'ont pas influencé de manière significative le rendement du manioc dans les conditions agro écologiques de M'vuazi, il serait intéressant de se pencher sur d'autres systèmes de culture, notamment le travail minimum du sol et le non labour avec paillage qui présentent plusieurs avantages notamment l'économie sur le coût du labour, la lutte contre l'érosion du sol et le contrôle des mauvaises herbes, la préservation de la teneur en eau du sol et de sa santé, la réduction de la quantité d'inputs extérieurs (FAO, 2013 ; Takahashi et Fidalski, 2019).

\section{Conclusion}

Cette étude a évalué l'effet de trois modes de labour : labour à plat $\left(\mathrm{T}_{0}\right)$, labour en billon $\left(\mathrm{T}_{1}\right)$ et labour en butte $\left(\mathrm{T}_{2}\right)$ sur le rendement du manioc. Les résultats ont montré qu'aucun traitement n'a amélioré de manière significative le nombre de racines tubéreuses par plante, le rendement en pulpe fraîche et le rendement en matière sèche. Toutefois, le labour en butte et en billon ont entrainé, respectivement, un manque à gagner de 88 et 288 \$US ha ${ }^{-1}$ par rapport au labour à plat. Ainsi, dans les conditions de notre expérimentation, le labour en billon ou en butte semble inopportun mais nécessite d'être testé sur différents types de sol et des terrains accidentés.

\section{CONFLIT D'INTERETS}

Les auteurs attestent qu'il n'existe aucun conflit d'intérêts entre eux ou autre partie au sujet de cet article.

\section{CONTRIBUTIONS DES AUTEURS}

TMM et EBN ont rédigé le protocole expérimental, ces deux auteurs et PMM, ont installé l'essai, EBN et PMM ont fait le suivi l'essai. Les 3 auteurs ont organisé la récolte et collecté les données de rendement, TMM et EBN ont fait la vérification et l'exploitation des données (analyses statistiques) ainsi que la rédaction de la première version du manuscrit.
TMM a veillé à la conformité des résultats et à la rédaction de la version finale du manuscrit.

\section{REMERCIEMENTS}

Les auteurs remercient les ouvriers du Programme National des Ressources Phytogénétiques du Centre de Recherche INERA M'vuazi qui ont assuré l'entretien de l'essai expérimental de cette étude.

\section{RÉFÉRENCES}

Agbede TM, Adekiya AO. 2009. Tillage effects on soil properties and performance of sweet potato on an Alfisol in Southwestern Nigeria. Am.-Eurasian J. Sustain. Agric., 3(3): 561-568.

Aiyellari E, Ndaeyo N, Agboola A. 2002. Effects of tillage practices on growth and yield of cassava (Manihot esculenta Crantz) and some soil properties in Ibadan, Southwestern Nigeria. Tropicultura, 20(1): 29-36.

Bilong E, Ngome F, Abossolo-Angue M, Birang M, Madong, Ndaka S, Bilong P. 2017. Effets des biomasses vertes de Tithonia diversifolia et des engrais minéraux sur la croissance, le développement et le rendement du manioc (Manihot esculenta Crantz) en zone forestière du Cameroun. Int. J. Biol. Chem. Sci., 11(4): 1716-1726. DOI: http://dx.doi.org/10.4314/ijbcs.v11i4.24

Denisoff I, Devred R. 1954. Cartes des sols et de la végétation du Congo Belge et du Ruanda-Urundi. 2. Mvuazi (Bas-Congo). Notice explicative de la carte des sols et de la végétation. Institut National pour l'Etude Agronomique du Congo Belge (I.N.E.A.C): Bruxelles.

FAO. 2014. Champs-Ecoles Paysans sur le Manioc. Ressources à l'Intention des Facilitateurs d'Afrique Sub-Saharienne. Publications de la FAO : Rome.

FAO. 2013. Produire plus avec moins: Le manioc. Guide pour une Intensification Durable de la Production. Publications de la FAO : Rome.

Fasinmirin TJ, Reichert JM. 2011. Conservation tillage for cassava (Manihot esculanta Crantz) production in tropics. 
Soil Till. Res., 113: 1-10. DOI: http://dx.doi.org.10.1016/j.still.2011.01.0 08 .

HarvestPlus. 2010. Provitamin A cassava in the Democratic Republic of Congo. HarvestPlus: Washington. 45p.

Kabongo JP, Pongi G, Mbuya A, Tshimbombo C, Kizungu R. 2019. Comparative study of maize agronomics performance under low nitrogen soil availability, in southern savanna of DR Congo. Afrika Focus, 32(2): 57-64. DOI: https://doi.org/10.21825/af.v32i2.15767.

Kintché K, Hauser S, Mahungu N, Ndonda A, Lukombo S, Nhamo N, Uzokwe VN, Yomeni M, Ngamitshara J, Ekoko B, Mbala M, Akem C, Pypers P, Matungulu KP, Kehbila A, Vanlauwe B. 2017. Cassava yield loss in farmer fields was mainly caused by low soil fertility and sub-optimal management practices in two provinces of the Democratic Republic of Congo. Eur. J. Agron., 89 : 107-123. DOI: https://doi.org/10.1016/j.eja.2017.06.011

Lamadi W. 2016. Effect of different tillage practices on cassava production in Osun State of Nigeria. Res. J. Agric. Environ. Manag., 5(4) : 114-121.

Mahungu N, Ndonda A, Frangoie N, Maongo M. 2015. Effet du labour et du mode de bouturage sur les rendements en racines et en feuilles de manioc dans les zones de savane et jachères forestières de la République démocratique du Congo. Tropicultura, 33(3): 176-185.

MINAGRI. 2012. Catalogue variétal des cultures vivrières : maïs, riz, haricot, soja, niébé, manioc, patate douce, pomme de terre, bananier. Service National de Semences : Kinshasa/RD Congo. 158p.

Mondo J, Irenge A, Ayagirwe R, Nguezet DP, Karume K, Njukwe E, Mapatano S, Zamukulu P, Basimine G, Musungayi E, Mbusa Héritier, Kazamwadi L, Civava R, Mushagalusa G. 2019. Determinants of adoption and farmer's perceptions preferences for cassava varieties in Kabare territory, Eastern Democratic Republic of Congo. Am. J. Rural Dev., 7(2): $\quad 44-52 . \quad$ DOI: http://pubs.sciepub.com/12691/ajrd/7/2/1

Muliele TM, Manzenza CM, Ekuke LW, Diaka CP, Ndikubwayo DM, Kapalay, OM, Mundele AN. 2018. Utilisation et gestion des pesticides en cultures maraîchères: cas de la zone de Nkolo dans la province du Kongo Central, République démocratique du Congo. J. Appl. Biosci., 119: 11954-11972. DOI: https://doi.org/10.4314/jab.v119i1.11.

Odjugo P. 2008a. The impact of tillage systems on soil microclimate, growth and yield of cassava (Manihot utilisima) in Midwestern Nigeria. Afr. J. Agric. Res., 3(3): 225-233.

Odjugo P. 2008b. The effect of tillage systems and mulching on soil microclimate, growth and yield of yellow yam (Dioscorea cayenensis) in Midwestern Nigeria. Afr. J. Biotechnol., 7(24): 45004507.

Takahashi M, Fidalski J. 2019. Soil tillage to produce cassava roots with less physical limitations in pasture succession. Sci. Agrar. Parana. 18(4): 383-388.

Temegne N, Ngome F, Fotso A. 2015. Influence de la composition chimique du sol sur la teneur en éléments nutritifs et le rendement du manioc (Manihot esculenta Crantz, Euphorbiaceae) dans deux zones agro-écologiques du Cameroun. Int. J. Biol. Chem. Sci. 9(6): 2776-2788. DOI: http://dx.doi.org/10.4314/ijbcs.v9i6.21.

Yuku E, Kimbembi I. 2018. Mise en œuvre d'un système de production durable de manioc (Manihot esculenta Crantz) dans le village de Tadila en République Démocratique du Congo. Tropicultura 36(4) : $641-648$. 